\title{
Analytical method for determining at equilibrium the envelope and emittance of initially mismatched beams
}

\author{
R. P. NUNES ${ }^{1}$ and F. B. R IZZA T O ${ }^{2}$ \\ ${ }^{1}$ CTI, Universidade Federal do Rio Grande, Rua Eng. Alfredo Huch 475, \\ CEP 96201-900, Rio Grande, RS, Brasil \\ (rogerpn@if.ufrgs.br, roger.pizzato@ufrgs.br) \\ ${ }^{2}$ Instituto de Física, Universidade Federal do Rio Grande do Sul, Av. Bento Gonçalves, \\ 9500, Caixa Postal 15051, CEP 91501-970, Porto Alegre, RS, Brasil
}

(Received 19 January 2009 and accepted 20 April 2009, first published online 3 June 2009)

\begin{abstract}
This work presents a fully analytic way of determining relevant equilibrium quantities of a high-intensity charged particle beam submitted to magnetic focusing while inside a linear channel. Through the current approach, some intermediate steps of our original hybrid model which had to be solved numerically can now be eliminated, leading to the obtainment of a fully analytic expression. This expression relates the initial beam parameters with those at equilibrium, allowing beam macroscopic quantities such as envelope and emittance to be naturally and analytically determined. For validation, full self-consistent $N$-particle beam numerical simulations have been carried out and the results compared with the predictions supplied by the full analytical model. The agreement is shown to be good with the simulations and also with the original hybrid numerical-analytical version of the model.
\end{abstract}

\section{Introduction}

As charged beams with mismatched envelopes evolve inside the focusing channel, a small number of particles are ejected, developing large amplitude orbits in phase space very different from those plasma-like orbits performed by the remaining particles. This is a natural consequence of the initial beam mismatch, which works as an energy source to the gradual excitation of individual particles. Although small and thus apparently not impacting in the calculations, the contribution of this rarefied particle population to the calculation of the statistically averaged beam quantities becomes more important as equilibrium approaches.

At equilibrium, observing the beam configuration space, it is possible to detect that the initially spatially limited beam particle population is at this time additionally surrounded by a tenuous particle population. From inspection of the beam phase space, it is easy to identify that the previously commented tenuous population is now dispersed in such a way that its influence over the entire beam cannot no longer be neglected. Particles pertaining to this population acquire so much energy that their state variables are very different from those of which the 


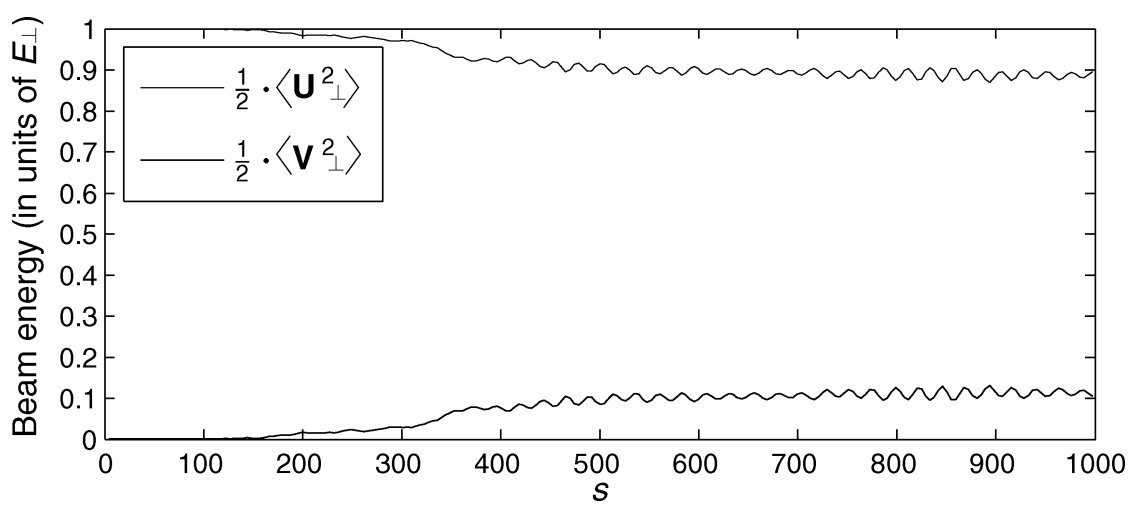

Figure 1. Dynamics of the beam potential and kinetic energies during its magnetic focusing inside the linear channel. Results provided by self-consistent beam numerical simulations of $N=10000$ particles. Beam initial mismatch $r_{0}=1.5$.

beam is composed. This tenuous population is commonly recognized as the halo in the beam physics field.

Halo formation is a phenomenon observed in self-consistent $N$-particle beam numerical simulations [1] as well as in experiments [2], and it has several implications affecting beam characteristics at equilibrium. In this way, halo formation has become a subject that is studied extensively in beam physics, and its effects on the infrastructure of the accelerator are a problem to be mitigated in its engineering.

Macroscopically, the above-mentioned particle ejection is perceived as the change of two statistically averaged quantities of the beam distribution during its excursion inside the focusing channel. One is the beam envelope, which suffers an important decay, and the other is the beam emittance, which experiences in addition growth that is not negligible. The beam envelope and emittance have an inverse dynamical behavior - the first decays and the second grows - not by coincidence, but because they are concatenated by a constraint: energy conservation.

The beam distribution evolves by assuming that its overall energy remains constant. In this way, if the beam envelope decays (inducing potential energy decreases), then the emittance must grow (imposing kinetic energy increases), because the overall beam energy is inevitably conserved. To illustrate the last point, the dynamics of the kinetic and potential beam energies are shown in Fig. 1, in which the results are obtained from self-consistent $N$-particle beam simulations of an initially mismatched beam. The way particles are numerically simulated will become clear in Sec. 2. The striking characteristic observed here, which will be discussed in Sec. 2, is that almost all the beam kinetic energy is carried by a small number of particles. Since emittance and kinetic energy are directly connected, the tenuous population has therefore great importance to the emittance growth during the focusing process.

This progressive increasing of beam kinetic energy is proportioned by the interaction of individual particles with the mismatched beam. As the beam propagates inside the focusing channel, its envelope mismatch induces the formation of large resonant islands [3] beyond its border in phase space. Individual particles are captured by these resonances [4], coupling their motion with the mismatched beam. The resonant coupling - an outcome of the particle-beam interaction - is the way that energy exchange occurs: the potential energy of the beam oscillatory motion 
(macroscopic energy) is progressively converted into kinetic energy that supplies the chaotic movement of the individual particles (microscopic energy). Equilibrium is reached when this energy transfer mechanism ceases. Together, all the other mentioned statistically averaged quantities also stabilize. At this point, it is of interest to know the values of the envelope and emittance achieved, not only for physics purposes but also for engineering aspects, since the halo characteristics are a factor to be considered in the design of accelerator confinement structures.

The purpose of the current article is to obtain a fully analytic expression for the model introduced and described in [5], which has many steps that unconditionally have to be solved numerically. In this way, not only can the beam envelope be evaluated as an analytical function of the only one free parameter in the model, the beam initial mismatch, but so can the beam emittance. Further discussion about the current subject is also provided. For simplification of the calculations, the beam has been considered to be azimuthally symmetric and initially homogeneous. For validation, the results provided by the full analytical expression have been compared with the ones provided by the numeric version of the model and the full self-consistent $N$-particle beam numerical simulations, considering several values of initial beam mismatch.

This article is arranged in the following form: in Sec. 2, important points of the model are formally presented. To simplify the calculation, the beam has been considered initially without emittance, i.e. a cold beam. In Sec. 3, the strategy developed to solve the model analytically is shown and the expression that relates the beam equilibrium state quantities with those at the beam initial state is presented. In Sec. 4, the results for beam envelope and emittance at equilibrium calculated through the full analytical approach are compared with the previous numeric version of the model and with the full self-consistent $N$-particle simulations. This comparison has been carried out for several values of initial beam mismatch. Finally, also in this section, the conclusions of this article and the perspectives for future works are discussed.

\section{The model}

The system of interest considered here is a high-intensity beam of charged particles, focused by a constant magnetic field during its evolution inside a linear propagation channel, encapsulated by a circular conducting pipe. Beam centroid oscillations are negligible, since perfect alignment with the symmetry axis of the confinement channel is conferred to the beam. Also, the beam is considered to be infinitely long in the longitudinal dimension front of the transversal dimension (the thin beam approximation).

For simplicity of the calculations, the initial beam density is considered to be homogeneous in the configuration space. Azimuthal symmetry is also assigned to the beam for convenience. Additionally, beam particles are initially halted, which means that a cold approximation is adequate for the beam initial state description. The initial representation of the beam in phase space is then irrelevant, it being sufficient just to define its spatial charge distribution. In this way, a step-function profile can be employed to model the beam initial state

$$
n(r, s=0)= \begin{cases}N / \pi r_{0}^{2} & \text { for } 0 \leqslant r \leqslant r_{0}, \\ 0 & \text { for } r_{0}<r \leqslant r_{\mathrm{w}},\end{cases}
$$



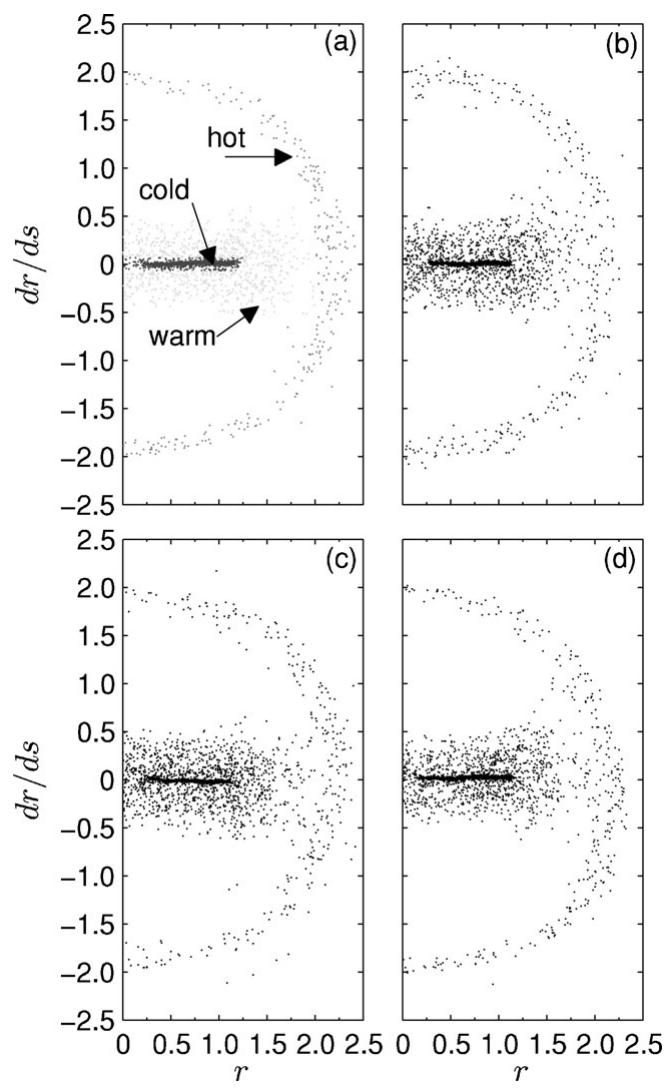

Figure 2. Snapshots of the beam transverse phase space at its equilibrium for an initial envelope mismatch of $r_{0}=1.5$ obtained with self-consistent $N$-particle beam numerical simulations. Phase-space topology invariance is attained. Phase-space portraits captured at (a) $s=547.8$, (b) $s=599.7$, (c) $s=798.7$ and (d) $s=997.6$.

where $N$ is the total number of particles of which the beam is composed, $r_{0}$ designates the initial beam mismatch and $r_{\mathrm{w}}$ is the conducting pipe location. The radial coordinate $r$ explores the beam transversal section and is in the Larmor frame, while the coordinate $s$ refers to the longitudinal beam path. Coordinate $s$ can also be recognized as time since both are trivially related by the expression $s=s_{0}+v_{\mathrm{b}} t$ in which $v_{\mathrm{b}}$ is the beam axial velocity, which is a constant. All transverse coordinates are rescaled in units of the beam equilibrium radius, defined a $r_{\text {eq }}=\sqrt{K / \kappa}$, and the longitudinal coordinates are rescaled in units of $1 / \sqrt{\kappa}$. This is exactly equivalent to setting

$$
K \rightarrow 1 \text { and } \kappa \rightarrow 1
$$

in all equations in which these parameters appear. The quantity $K$ is the beam perveance and $\kappa$ is the coefficient of magnetic confinement. Both are constant in the context of this work.

In Fig. 2, several snapshots of the beam transverse phase space after equilibrium is attained are shown. Here, self-consistent $N$-particle beam simulations using Gauss's Law have been performed. A simulation method through Gauss's Law is suitable here once collective effects only are accounted for, since particle binary 


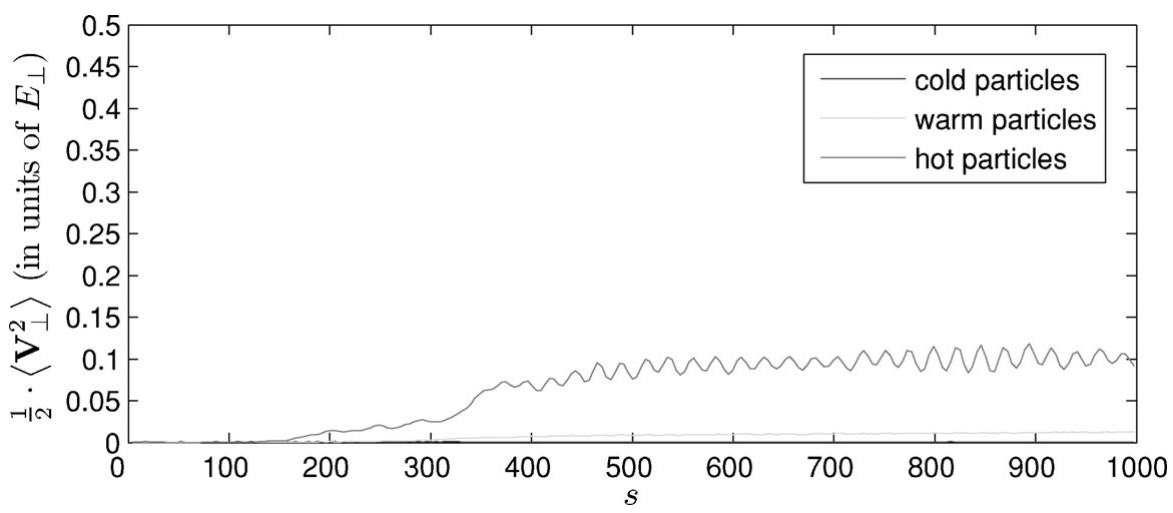

Figure 3. The population classifications and their kinetic energy at any instant $s$ of the beam dynamics inside the linear focusing channel. Results provided by self-consistent beam numerical simulations of $N=10000$ particles. Beam initial mismatch $r_{0}=1.5$.

encounters are nonexistent: beam individual particles just interact with the other particles by self-consistent and self-generated electromagnetic fields resulting from the dynamics of the beam charge density [1]. A total of $N=10000$ particles have been employed for convergence. The initial beam mismatch is $r_{0}=1.5$.

It is possible to observe in Fig. 2 that the equilibrium is directly associated to the invariance of the phase-space topology. Particle orbits are defined in such a way that they occupy a well-defined and limited region in phase space. In each of these regions, the number of particles remains almost constant. This is the means by which statistically averaged quantities of the beam distribution stabilize at equilibrium. On average, the microscopic states (composed of the coordinates of each particle in phase space) do not considerably change. Since macroscopic quantities are just averages over the microscopic states, these quantities do not experience any alteration in their values. In this case the beam quantities such as envelope and emittance are included.

The regular aspect of the beam phase space at equilibrium allows its structure to be decomposed into three distinct regions: a horizontal thin branch, composed of very cold particles; a cloud around the horizontal branch, made up of warm particles; and a curve branch, populated by extremely hot particles. In Fig. 2(a), the cold, warm and hot particles are shown. Although warm particles have a fundamental role in halo formation, thus important for the description of the beam transient behavior [6], their contribution at equilibrium can be neglected. In fact, warm particles store much less than $10 \%$ of the total beam kinetic energy at equilibrium. This can be seen in Fig. 3, in which the dynamical behavior of the beam kinetic energy is decomposed in each of the classifications above and accounted for in units of the total beam transverse energy $E_{\perp}$. Observe that the kinetic energy curve of the hot particles in Fig. 3 is almost equal to the curve of the total kinetic energy in Fig. 1. In this sense, as an approximation, the velocity of warm particles can be assumed to be unimportant at equilibrium, being considered as cold particles [6]. For the current mismatch, the total transversal beam energy is assumed to be $E_{\perp}\left(r_{0}=1.5\right)=0.4843$. Note that the particular kinetic energy of each particle is unimportant here. Discrimination just occurs for populations, since every beam macroscopic quantity is obtained from averages over its distribution. 
This approximation allows reclassification of particles in a dichotomic way: it is possible to associate the cold particles to the beam core as well as the hot particles to those that make up the beam halo.

The regular geometry of each previously mentioned region of the beam phase space can be directly converted to an analytical expression. At equilibrium, the beam density can be segmented in the form [5]

$$
n(r, s \geqslant \tau)= \begin{cases}n_{\mathrm{c}}(r)+n_{\mathrm{h}}(r) & \text { for } 0 \leqslant r \leqslant r_{\mathrm{c}} \\ n_{\mathrm{h}}(r) & \text { for } r_{\mathrm{c}}<r \leqslant r_{\mathrm{h}}, \\ 0 & \text { for } r_{\mathrm{h}}<r \leqslant r_{\mathrm{w}}\end{cases}
$$

where $n_{\mathrm{c}}$ and $n_{\mathrm{h}}$ are, respectively, the particle density of the beam core and the beam halo. The quantity $r_{\mathrm{c}}$ is the core size and $r_{\mathrm{h}}$ is associated with the size projected by the curve branch over the $r$-axis. The variable $\tau$ represents the time scale in which equilibrium is reached.

Since the core particles at equilibrium have characteristics that are remnant of the initial state, this population can still be represented as a step-function profile [5]

$$
n_{\mathrm{c}}(r)=(1-f) N / \pi r_{\mathrm{c}}^{2},
$$

but now only with $r_{\mathrm{c}}<r_{0}$ and a smaller number of particles given by $N_{\mathrm{c}}$, expressed formally by the fraction of halo particles $f \equiv N_{\mathrm{h}} / N$ through the particle conservation relation $N=N_{\mathrm{c}}+N_{\mathrm{h}}$.

The halo population pertaining to the curve branch in a semicircular approximation follows [7]

$$
n_{\mathrm{h}}(r)=\frac{f N}{\pi^{2} r \sqrt{r_{\mathrm{h}}^{2}-r^{2}}} .
$$

Note that the halo population is not homogeneous, as it is described by an irrational function with a clear divergence at $r=r_{\mathrm{h}}$. This is a natural consequence of the geometry of the resonance, whose formation is naturally induced by the initial beam mismatch.

Both (4) and (5) completely define (3). Since (3) contains all the spatial information about the beam at equilibrium, one thus becomes able to evaluate at this state any of its macroscopic quantities as a function of the fraction of the halo particles $f$, which remains undetermined. Nevertheless, observe that this quantity can be directly determined, because the overall beam energy stored at equilibrium must be the same as that calculated at the initial state.

With the beam density resolved at the initial state and at equilibrium, it is possible to determine its self-consistent generated electric field $\mathbf{E}$ by means of the following Maxwell equation [8]:

$$
\nabla \cdot \mathbf{E}=-\frac{2 \pi K}{N} n(r, s)
$$

Solving this Maxwell equation with the aid of (1) and (3), one respectively obtains the expressions for $\mathbf{E}$ at the initial beam state,

$$
E_{r}(r, s=0)= \begin{cases}r / r_{0}^{2} & \text { for } r \leqslant r_{0} \\ 1 / r & \text { for } r_{0}<r \leqslant r_{\mathrm{w}}\end{cases}
$$


and the expression for $\mathbf{E}$ at equilibrium,

$$
E_{r}(r, s \geqslant \tau)= \begin{cases}-\frac{(1-f) r}{r_{\mathrm{c}}^{2}}-\frac{2 f}{\pi r} \arctan \left(\frac{r}{\sqrt{r_{\mathrm{h}}^{2}-r^{2}}}\right) & \text { for } r \leqslant r_{\mathrm{c}}, \\ -\frac{2 f}{\pi r} \arctan \left(\frac{r}{\sqrt{r_{\mathrm{h}}^{2}-r^{2}}}\right)-\frac{(1-f)}{r} & \text { for } r_{\mathrm{c}}<r \leqslant r_{\mathrm{h}}, \\ -\frac{1}{r} & \text { for } r_{\mathrm{h}}<r \leqslant r_{\mathrm{w}} .\end{cases}
$$

The overall beam energy can be computed at any instant of time $s$ through

$$
\kappa \frac{r_{b}^{2}(s)}{4}+\frac{\left\langle\mathbf{V}_{\perp}^{2}\right\rangle(s)}{2}+\varepsilon(s)=E_{\perp}=\text { constant }
$$

in which the average self-field beam energy [8] is formally given by

$$
\varepsilon(s)=\frac{1}{4 \pi K} \int|\mathbf{E}|^{2} d \mathbf{r},
$$

the average kinetic energy has the form, for any $s$,

$$
\left\langle\mathbf{V}_{\perp}^{2}\right\rangle(s) \approx \begin{cases}0 & \text { for } 0 \leqslant s \lesssim \tau, \\ \frac{r_{b}^{2}(s \geqslant \tau)-1}{2} & \text { for } s \gtrsim \tau,\end{cases}
$$

and finally the beam envelope $r_{\mathrm{b}}$ assumes at the initial state and equilibrium the simple expression [5]

$$
r_{\mathrm{b}}^{2}(s) \approx \begin{cases}r_{0}^{2} & \text { for } 0 \leqslant s \lesssim \tau, \\ (1-f) r_{\mathrm{c}}^{2}+f r_{\mathrm{h}}^{2} & \text { for } s \gtrsim \tau .\end{cases}
$$

As a result of energy conservation, the beam emittance can also be characterized by the following expression [5]:

$$
\varepsilon^{2}(s) \approx \begin{cases}0 & \text { for } 0 \leqslant s \lesssim \tau, \\ r_{\mathrm{b}}^{2}\left(r_{\mathrm{b}}^{2}-1\right) & \text { for } s \gtrsim \tau .\end{cases}
$$

Equations (11), (12) and (13) in fact are an approximation. The envelope decay observed during the excursion of the beam inside the focusing channel is not really a discontinuous function of axial coordinate $s$. Yet, this approximation becomes better as initial beam mismatch $r_{0}$ increases. However, this is not a problem in this work, since our interest resides on just two specific times for the beam dynamics, $s=0$ and $s \geqslant \tau$.

Inserting (7), (11) and (12) into (9), the beam energy at the initial state is obtained. Proceeding in the same way, just considering (8) for the self-consistent electric field, the expression for the beam transversal energy $E_{\perp}$ at equilibrium is obtained. Making use of energy conservation, equating

$$
E_{\perp}(s=0)=E_{\perp}(s \geqslant \tau),
$$

it becomes possible to generate a closed expression for the fraction of halo particles $f$. This equation is a second-order polynomial [5] with the form

$$
A f^{2}+B f+C=0,
$$




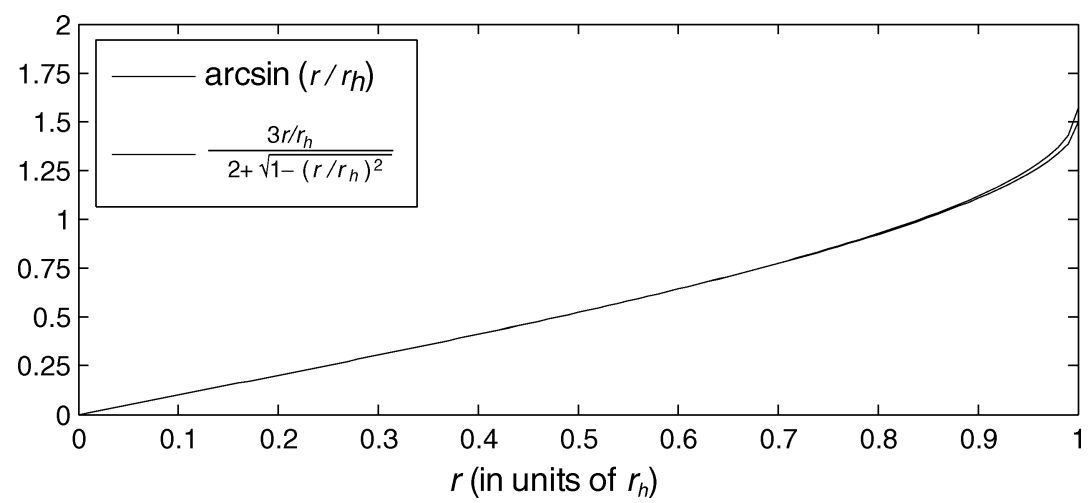

Figure 4. Comparison between the inverse sine function and its approximation adopted to solve analytically the integrals involving complicated electric field expressions at equilibrium.

in which $A, B$ and $C$ are integral functions of the beam phase-space parameters $r_{0}, r_{\mathrm{c}}$ and $r_{\mathrm{h}}$. In principle these coefficients have to be solved numerically. Physical meaning imposes that the desired solution resides between $0 \leqslant f \leqslant 1$.

\section{The analytical solution}

As (9) shows, the average self-field beam energy at any instant of time $s$ depends on the square value of the electric field. For this reason, due to the complexity of the electric field expressions shown in (8), at equilibrium an exact analytical solution to the coefficients, principally for $A$ and $B$, of the polynomial in (15) is difficult if not impossible. Nevertheless, recognizing that

$$
\arctan \left(\frac{r}{\sqrt{r_{\mathrm{h}}^{2}-r^{2}}}\right)=\arcsin \left(\frac{r}{r_{\mathrm{h}}}\right),
$$

the following approximation for the arc sine can be adopted [9]:

$$
\arcsin \left(\frac{r}{r_{\mathrm{h}}}\right) \approx \frac{3\left(r / r_{\mathrm{h}}\right)}{2+\sqrt{1-\left(r / r_{\mathrm{h}}\right)^{2}}},
$$

that is, the original are tangent function has been converted to an irrational one that can now be integrated analytically. Comparison between the arc sine function and its approximation in (17) is shown in Fig. 4. There is a visible discrepancy between both expressions as $r / r_{\mathrm{h}} \rightarrow 1$.

Inserting (17) into (8) gives the following approximation to the self-consistent electric field $\mathbf{E}$ at equilibrium:

$$
E_{r}(r, s \geqslant \tau) \approx \begin{cases}-\frac{(1-f) r}{r_{\mathrm{c}}^{2}}-\frac{6 f / \pi}{2 / r_{\mathrm{h}}+\sqrt{r_{\mathrm{h}}^{2}-r^{2}}} & \text { for } r \leqslant r_{\mathrm{c}}, \\ -\frac{6 f / \pi}{2 / r_{\mathrm{h}}+\sqrt{r_{\mathrm{h}}^{2}-r^{2}}-\frac{(1-f)}{r}} & \text { for } r_{\mathrm{c}}<r \leqslant r_{\mathrm{h}}, \\ -\frac{1}{r} & \text { for } r_{\mathrm{h}}<r \leqslant r_{\mathrm{w}} .\end{cases}
$$


After exhaustive algebra the coefficients $A, B$ and $C$ of the polynomial in (15) can be obtained [6] providing through (18) the following expressions:

$$
\begin{aligned}
& A\left(r_{\mathrm{c}}, r_{\mathrm{h}}\right) \approx \varsigma+\ln \left(r_{\mathrm{c}}^{2} / r_{\mathrm{h}}^{2}\right)-[72 \ln (2 / 3)+24] / \pi^{2}-1 / 2, \\
& B\left(r_{\mathrm{c}}, r_{\mathrm{h}}\right) \approx-\varsigma+2 \ln \left(r_{\mathrm{c}}^{2} / r_{\mathrm{h}}^{2}\right)+1+2\left(r_{\mathrm{c}}^{2}-r_{\mathrm{h}}^{2}\right), \\
& C\left(r_{0}, r_{\mathrm{c}}\right) \approx 1-2 r_{\mathrm{c}}^{2}+r_{0}^{2}+\ln \left(r_{\mathrm{c}}^{2} / r_{0}^{2}\right) .
\end{aligned}
$$

Here $\varsigma$ is the auxiliary equation

$$
\begin{aligned}
\varsigma\left(r_{\mathrm{c}}, r_{\mathrm{h}}\right)= & -\left(\frac{48 r_{\mathrm{h}}^{2} \sqrt{3}}{\pi r_{\mathrm{c}}^{2}}+\frac{16 \sqrt{3}}{\pi}\right) \arctan \left[\frac{\sqrt{3} r_{\mathrm{h}}}{3 r_{\mathrm{c}}}\left(1+2 \sqrt{1-\frac{r_{\mathrm{c}}^{2}}{r_{\mathrm{h}}^{2}}}\right)\right] \\
& +\left(\frac{84 r_{\mathrm{h}}^{2}}{r_{\mathrm{c}}^{2}}+\frac{24}{\pi}\right) \arcsin \left(\sqrt{1-\frac{r_{\mathrm{c}}^{2}}{r_{\mathrm{h}}^{2}}}\right)-\frac{48-12 \sqrt{1-\left(r_{\mathrm{c}}^{2} / r_{\mathrm{h}}^{2}\right)}}{\pi r_{\mathrm{c}} / r_{\mathrm{h}}} \\
& +\frac{24 \sqrt{3}-42}{r_{\mathrm{c}}^{2} / r_{\mathrm{h}}^{2}}+\frac{8 \sqrt{3}}{2} .
\end{aligned}
$$

However, the quantities of interest here are the beam envelope $r_{\mathrm{b}}$ and the beam emittance $\varepsilon$ at equilibrium. In this way, it is possible to implement a change of variables in (15), expressing the fraction of halo particles $f$ through $r_{\mathrm{b}}$ or $\varepsilon$. From (12), isolating fraction $f$ one obtains

$$
f=\frac{r_{\mathrm{c}}^{2}-r_{\mathrm{b}}^{2}}{r_{\mathrm{c}}^{2}-r_{\mathrm{h}}^{2}} .
$$

Inserting (21) above into (15) and collecting terms for powers of $r_{\mathrm{b}}$,

$$
A^{\prime} r_{\mathrm{b}}^{4}+B^{\prime} r_{\mathrm{b}}^{2}+C^{\prime}=0
$$

in which the coefficients of the above polynomial are now described by

$$
\begin{aligned}
A^{\prime} & =\frac{A}{\left(r_{\mathrm{c}}^{2}-r_{\mathrm{h}}^{2}\right)^{2}}, \\
B^{\prime} & =-\frac{B}{r_{\mathrm{c}}^{2}-r_{\mathrm{h}}^{2}}-\frac{2 A r_{\mathrm{c}}^{2}}{\left(r_{\mathrm{c}}^{2}-r_{\mathrm{h}}^{2}\right)^{2}} \text { and } \\
C^{\prime} & =C+\frac{B r_{\mathrm{c}}^{2}}{r_{\mathrm{c}}^{2}-r_{\mathrm{h}}^{2}}+\frac{A r_{\mathrm{c}}^{4}}{\left(r_{\mathrm{c}}^{2}-r_{\mathrm{h}}^{2}\right)^{2}},
\end{aligned}
$$

that is, coefficients $A^{\prime}, B^{\prime}$ and $C^{\prime}$ can be readily obtained since they are just functions of the coefficients $A, B$ and $C$ properly defined in (19) and known quantities of the beam phase space at the initial state and at equilibrium.

In summary, to obtain the beam envelope $r_{\mathrm{b}}$ and the beam emittance $\varepsilon$ at equilibrium the following steps are needed.

(1) Once $r_{\mathrm{c}}$ and $r_{\mathrm{h}}$ are known parameters, compute the coefficients $A, B$ and $C$ with the aid of (19) and (20).

(2) Employ (23) to determine coefficients $A^{\prime}, B^{\prime}$ and $C^{\prime}$ using the results of step 1. 
Table 1. The results obtained through the developed model (numerical and quasi-exact analytical solutions) and their comparison with those calculated from the full self-consistent $N$-particle beam numerical simulations.

\begin{tabular}{ccccccc}
\hline & $r_{0}=1.0$ & $r_{0}=1.2$ & $r_{0}=1.4$ & $r_{0}=1.6$ & $r_{0}=1.8$ & $r_{0}=2.0$ \\
\hline$r_{\mathrm{c}}$ & $=1$ & $\cong 1.05$ & $\cong 1.10$ & $\cong 1.10$ & $\cong 1.20$ & $\cong 1.20$ \\
$r_{\mathrm{n}}$ & $=0$ & $\cong 1.68$ & $\cong 1.88$ & $\cong 2.00$ & $\cong 2.13$ & $\cong 2.25$ \\
& $=1$ & $\cong 1.03474$ & $\cong 1.11179$ & $\cong 1.21944$ & $\cong 1.33770$ & $\cong 1.46636$ \\
$r_{\mathrm{b}}$ & $=0$ & $\cong 0.27512$ & $\cong 0.54021$ & $\cong 0.85104$ & $\cong 1.18855$ & $\cong 1.57264$ \\
$\epsilon$ & $=1$ & Semicircular approximation - analytical solution & \\
$r_{\mathrm{b}}$ & $=1$ & $\cong 1.03475$ & $\cong 1.11194$ & $\cong 1.21967$ & $\cong 1.33801$ & $\cong 1.46672$ \\
$\epsilon$ & $=0$ & $\cong 0.27515$ & $\cong 0.54067$ & $\cong 0.85168$ & $\cong 1.18946$ & $\cong 1.573773$ \\
& \multicolumn{7}{c}{ Self-consistent numerical simulations } \\
$r_{\mathrm{b}}$ & $=1$ & $\cong 1.02893$ & $\cong 1.08063$ & $\cong 1.16717$ & $\cong 1.28389$ & $\cong 1.40507$ \\
$\epsilon$ & $=0$ & $\cong 0.23535$ & $\cong 0.45312$ & $\cong 0.76491$ & $\cong 1.12057$ & $\cong 1.50450$ \\
\hline
\end{tabular}

(3) Make use of the coefficients $A^{\prime}, B^{\prime}$ and $C^{\prime}$ to solve the polynomial in (22). Look for only one positive root.

(4) Use (13) to compute the emittance $\varepsilon$ at equilibrium, by recovering the previous result for the beam envelope $r_{\mathrm{b}}$ as calculated in step 3 .

\section{Results, discussions and future works}

The results obtained with the model through both the numerical and the analytical solutions presented in Sec. 3 are shown in Table 1. The results in the table have been calculated by employing the algorithm presented at the end of Sec. 3. Also, the results provided by the full self-consistent $N$-particle beam numerical simulations are shown. Comparison of the results occurs for the beam envelope $r_{b}$ and the beam emittance $\varepsilon$ at equilibrium. The rows show the results for beam quantities at equilibrium while the columns show each analyzed mismatch $r_{0}$, which comprises the cases of $r_{0}=\{1,1.2,1.4,1.6,1.8,2.0\}$. The first two rows show the beam phasespace parameters at equilibrium, which are necessary to evaluate the previously mentioned beam quantities. They are input into the algorithm described at the end of Sec. 3 .

Analyzing Table 1, it can be perceived that the results obtained from the full analytical model are almost the same as those computed by the direct numerical solution of (22). Also, the results provided by the model are very similar to those obtained from the self-consistent $N$-particle beam numerical simulations. This occurs for all the mismatch cases analyzed in this work.

Note that as a matter of fact the analytical solution presented in Sec. 3 is not completely exact. Approximations to the electric fields at equilibrium had to be adopted to ensure that energy stored in these fields could be analytically evaluated. Due to the good agreement with previous results, the expression found is therefore a quasi-exact solution to the problem.

In future publications, results involving an initial non-homogeneous beam will be presented. To this end, all methodology developed in this and in previous works will be extended to a beam that is spatially parabolic at the initial state. The results 
partially show that a nice description of this kind of beam at equilibrium can also be acquired.

\section{Acknowledgements}

This scientific research has been carried out with the aid of the National Centre of Supercomputation of the Brazil's South Region (CESUP), located at the Federal University of Rio Grande do Sul (UFRGS).

Also, the author Roger Pizzato Nunes thanks Dr Jorge Rodolfo Silva Zabadal, from the Department of Nuclear Engineering (DENUC) at UFRGS, for fruitful discussions about symbolic mathematic software tools.

Finally, this work has received financial support from the National Council for Scientific and Technological Development (CNPq), from the Fundação de Amparo à Pesquisa do Estado do Rio Grande do Sul (FAPERGS), both from Brazil, and from the Air Force Office of Scientific Research (AFOSR), USA, under grant No FA9550-06-1- 0345.

\section{References}

[1] Nunes, R. P., Pakter, R. and Rizzato, F. B. 2007 Phys. Plasmas 14(2), 023104.

[2] Allen, C. K. et al. 2002 Phys. Rev. Lett. 89(21).

[3] Lichtenberg, A. J. and Lieberman, M. A. 1992 Regular and Stochastic Motion. New York: Springer-Verlag, p. 115.

[4] Gluckstern, R. L. 1994 Phys. Rev. Lett. 73, 1247.

[5] Nunes, R. P., Pakter, R. and Rizzato, F. B. 2008 J. Appl. Phys. 104(1).

[6] Nunes, R. P. 2008 Technical Report, UFRGS.

[7] Nunes, R. P. and Rizzato, F. B. 2008 Proceedings of 11th European Particle Accelerator Conference, Genoa, Italy. Mulhouse: EPS-AG, p. 3206.

[8] Davidson, R. C. and Qin, H. 2001 Physics of Intense Charged Particle Beams in High Energy Accelerators. Singapore: World Scientific, p. 269 (Energy Conservation).

[9] Benammar, M. 2005 IEEE Transactions on Circuits and Systems 52(2), 263. 